\title{
UROLOGICAL EMERGENCIES
}

\author{
By John Sandrey, M.B., Ch.M., F.R.C.S. \\ Surgeon, St. Peter's Hospital; Consultant Urologist to the Royal Nary.
}

With the exception of acute retention of urine, urological emergencies are not common enough for any medical man, unless he specializes in this type of work, to become at all familiar with them. This seems to be the main reason why so many mistakes are made in diagnosis and treatment. From the point of view of treatment urological emergencies can be classified under three main headings.

I. True surgical emergencies which require immediate operative treatment. These include ruptures of the ureter, bladder or urethra, spontaneous perirenal haematoma, genital torsions of various kinds, extravasation of urine, acute pyonephrosis, acute paraphimosis and constriction of the penis from any other cause.

2. Where a symptom, such as acute retention of urine or severe and prolonged renal colic, takes precedence over the underlying cause. 'Though not surgical emergencies in the strict sense of the word, it is, nevertheless, correct to say that their proper management from the beginning will play a vital role in the patient's ultimate recovery. Preliminary measures to afford relief of what is, in the first instance, an urgent and painful condition will often become part of a planned attack on the underlying cause later on. In some cases the main symptom alone is relieved, the underlying cause being dealt with at some future date (e.g. when suprapubic drainage is required to relieve retention of urine due to an impassible urethral stricture). In others both cause and effect can be efficiently dealt with simultaneously, as when immediate prostatectomy or continuous dilatation of a narrow urethral stricture are carried out in certain cases of acute retention.

3. A group of acute conditions which are usually treated conservatively in the first place although operative treatment may eventually be needed. These conditions include the anurias, the more severe forms of renal haematuria and most contusions of the kidney and the external genitalia.

It will be appreciated that only the salient clinical features and more important principles of treatment can be indicated in this short review, and omissions are inevitable. The conditions found in Groups I and 3 are usually the surgeon's responsibility, but the role of the family doctor in early diagnosis and in eliminating delay in transferring the patient to hospital is perhaps just as important. In Group 2 the general practitioner will often institute and sometimes complete treatment in the patient's home. He should, therefore, be familiar with the management of such cases and provide himself with the proper equipment needed to deal with them efficiently.

Acute retention of urine is a relatively commonplace emergency which can generally be relieved promptly by catheterization in the patient's home difficulties only arising as a rule when severe obstructions at the bladder neck or in the urethra are encountered. As many of the patients in this category are elderly and in poor physical condition, from the effects of longstanding urinary obstruction, infection or intercurrent cardiovascular disease, it is most essential that they be given efficient treatment from the start. Catheterization, a simple enough procedure in most instances, may under certain circumstances become a harrowing experience for both practitioner and patient, and the number of admissions to hospital of patients with clot retention, false passages, urinary infections and unnecessary suprapubic cystotomies bears witness to the difficulties sometimes encountered.

No practitioner need ever suffer the humiliation of being unable to give prompt relief to a patient with a painful distended bladder if he carries a few relatively simple items of equipment in readiness for this type of emergency. They consist of :

One or two catheters of the Tieman pattern (sizes 14 and 16 charrière).

A fine lumbar puncture needle (Howard Jones' or similar pattern).

Several filiform bougies.

A tapered meatal dilator.

A medium-sized metal bougie for pushing 
foreign bodies or calculi impacted in the urethra back into the biadder.

These can all be sterilized quite simply in the home by boiling in any large dish or saucepan. Tieman's catheter (Fig. I) has many advantages over the gum-elastic instruments generally employed; it can be readily sterilized by boiling and its upturned, tapered and flexible end enables it to negotiate a distorted posterior urethra with ease, whereas a more rigid gum-elastic instrument will often be held up and may readily cause trauma and false passages if force is used.

If the patient is found to be distressed an injection of morphia on arrival at the house will allay anxiety and enable arrangements to be made for catheterization under proper aseptic conditions. Furthermore a quiet relaxed patient will be more co-operative than one who is restless and apprehensive. Intravenous pethidine ( 50 to $100 \mathrm{mg}$.) may be a boon when dealing with refractory patients.

Preliminary questioning may yield valuable information; for instance, the patient may be aware of the fact that he has a stricture or else may admit to having recently introduced a foreign body along the urethra. He may perhaps describe what is obviously a recent attack of renal colic, thus indicating the possibility of a calculus obstructing the urethra.

Examination of the patient will not only confirm that the bladder is distended but may sometimes bring other important facts to light, for example a meatal stricture or a perineal abscess, if present, will be obvious on external examination.

Where the cause is not apparent, an attempt should be made to pass a Tieman's catheter. If this is successful the prostate and bladder base should be carefully palpated per rectum after the bladder has been completely emptied and before the catheter is removed. In most cases a smooth bi-lobed enlargement of the prostate gland will be readily appreciated, and this type of case should be sent to hospital where, if conditions are favourable and the patient a 'good risk,' immediate prostatectomy is generally regarded as the best form of treatment. About 20 per cent. of all prostatic obstructions, however, are due to malignant disease. In such cases the gland is found to be stony hard, irregular and its borders ill-defined; the catheter should then be tied in for a week and full doses of stilboestrol ( 30 to $60 \mathrm{mg}$. daily) administered. The 'boggy' sensation of a prostatic abscess may be detected and when such a collection is at all large, adequate drainage from the perineum must be provided.

When a urethral stricture is present the passage of the catheter is obstructed in the bulb, usually Io to $12 \mathrm{~cm}$. from the external meatus. Some-

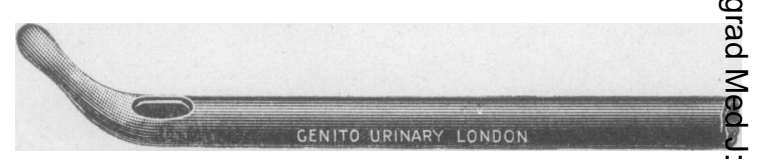

Fig. 1.-Tieman's cathetcr.

$\vec{F}$
$\stackrel{9}{+}$
음
음

times the stricture is of ' wide bore,' the attack $\frac{\text { क्न }}{7}$ retention being due to congestion from alcohow cold, etc. In these cases a small Tieman catheter will usually pass with ease. Generally, however, the stricture is contracted and unp yielding and, in these, infinite patience and gentle ness will be necessary to coax the finest filiforrfy bougie through its narrow lumen. When this successful urine will pass drop by drop alongside the instrument. After retention has been relieveis continuous dilatation of the stricture can be carrieg out by substituting larger and larger instruments This type of retention may be complicated by is periurethral abscess or by gangrenous cellulitis of the perineum. A profound toxaemia, rapidly fatat in untreated cases, usually accompanies the more severe forms of suppuration in this area. Treat ment, by free incision of the affected subcutaneous tissues, urinary diversion by perineal or suprapubi遥 cystostomy and chemotherapy, is urgently quired in these cases.

Suprapubic puncture is an alternative met when catheterization fails. It is also sometime? employed as a routine procedure, in cases of simple prostatic obstruction in order to avoid the risk of catheter infection, by those who practise thथ्๊ Wilson Hey technique of immediate " aseptic 8 prostatectomy in such circumstances. The punc $\overrightarrow{\overline{0}}$ ture is made with a very fine spinal needle im 3 mediately above the symphysis pubis and the bladder emptied as completely as possible. IR order to prevent leakage of urine from the puncture into the prevesical space the stylet should be re inserted immediately before withdrawal and the site of puncture firmly compressed for a fetr. moments after removal of the needle. The dangers of prevesical cellulitis following this method are greatly increased when the urine is heavily infected. If this complication is feared arrangements should be made to provide more adequate suprapubic drainage with the least possible delay. Injuries to the peritoneum or gut are rare and are only likely in very obese patients

Certain types of retention require specials treatment.

\section{Clot Retention}

Clot retention, where the outlet of the bladde $\frac{0}{\mathrm{~T}}$ is blocked by blood-clot, may follow urethra trauma or may be spontaneous, the latter being due as a rule to a vesical neoplasm. Evacuation of 
the clot through a large metal catheter by means of a syringe or Bigelow's evacuator is usually successful, but cystoscopic diathermy to a bleeding bladder growth or suprapubic cystostomy may be necessary to control severe haemorrhage.

\section{In Women}

In women, retention of urine is commonly due to urethral stricture, neoplasms infiltrating or prolapsing through the internal urinary meatus, foreign bodies or sometimes hysteria. Uterine enlargements such as fibroids or retroverted gravid uteri are, in the experience of the writer, very seldom the cause of acute retention of urine. Urethral stricture is a disease by no means confined to the male sex and is often observed in women after urethral trauma or infection. The condition is usually easy to treat by dilatation.

\section{Non-Obstructive Retention}

Non-obstructive retention may follow pelvic operations or confinements (' reflex retention ') or is observed in association with various neuropsychiatric disorders such as hysteria, arteriosclerosis, schizophrenia, etc. ('inhibitory retention '). The former state is painful, the latter is not. These conditions usually respond to parasympathetic . stimulants, and rarely require catheterization.

\section{Anuria}

Anuria can readily be distinguished from acute retention of urine by catheterization and finding an empty bladder. Failure to take this elementary precaution at the earliest possible moment in comatose or semi-comatose patients has often been responsible for avoidable delay in instituting treatment. Early recognition of the anuric state is of the utmost importance. Any patient who, after a severe operation, haemorrhage, abortion or crush injury, passes less than 500 cc. of urine in 24 hours must be kept under the closest scrutiny.

A wide variety of conditions, many of them primarily non-renal, can give rise to oliguria or anuria. Among them may be mentioned the crush syndrome, 'transfusion kidney,' various anuric syndromes associated with pregnancy and abortion, sulphonamide anuria, poisoning with heavy metals, anuria following shock, haemorrhage or haemolysis. The bedside of the anuric patient is, in fact, now the meeting place of nearly every type of specialist, all interested in different aspects of a many-sided problem.

During the past decade the important observations of Bywaters and other British workers on the crush syndrome and of Trueta and his associates on renal vascular 'shunts' have stimulated immense interest in renal physiology, both normal and abnormal, with the result that our conception of renal failure has had to undergo profound modification. Many of the loose descriptive terms formerly applied to these conditions, such as ' uraemia,' 'pre- or post-renal anuria,' have had to be abandoned and a more workable classification, based on the part of the nephron most affected, is now being universally adopted. Changes taking place in the nephron are found to be concentrated at three levels; the glomerular arterioles, the proximal renal tubules and the distal renal tubules:-

I. Firstly, cortical ischaemia from vasospasm may be observed when the blood pressure is greatly lowered, as in shock or cardiac failure, when the blood volume is diminished by haemorrhage, as the result of nervous stimuli (" reflex anuria') and possibly from toxaemia. Here the kidney itself is not necessarily diseased.

2. In the second group the brunt of the damage is observed to fall on the proximal tubules (" upper nephron nephrosis ') with marked impairment of their function. Degenerative changes at this level are observed in cases of renal toxaemia, eclampsia and poisoning by heavy metals.

3. In this group the distal tubules are chiefly affected ('lower nephron nephrosis ') and the main factor is obstructive. This obstruction occurs either in the distal tubules, by casts, heme pigment or acetyl sulphonamide, or in the collecting tubules which are dilated by back pressure from an obstruction at a lower level such as a ureteric calculus, bladder neck obstruction, etc.

Cases of anuria should be investigated very fully in order to classify them correctly at the earliest possible moment. This is by no means easy since the groups may overlap; for instance, sulphonamide anuria is often partly obstructive and partly toxic. In cases in the first and third groups the changes are often reversible and many recover with correct treatment. On the other hand permanent damage to the epithelium of the proximal tubules from longstanding renal disease is likely in the second group and in these anuria is a terminal event.

\section{Management}

The management of all cases of anuria, unless the condition is obviously a terminal event, must be based on the assumption that the renal damage is reversible. The objects of treatment are to maintain normal water balance, electrolyte, blood pressure, blood volume and haemoglobin levels, acid-base equilibrium and general nutrition. Attempts have also been made to remove excess of certain products of metabolism by means of peritoneal dialysis or the artificial kidney with varying degrees of success. A high calorie, 
protein-free diet, as recommended by Bull, Joekes and Lowe (1949), is administered by continuous drip through an indwelling gastric tube, any vomitus being filtered and returned to the stomach. The daily requirements of the average patient are satisfied by the following formula:-

Glucose

Peanut oil

Acacia, q.s. to emulsify

Water to 1 1. (Vitamins can be added if required.)

Surgical measures include catheterization of obstructed ureters and lavage of the renal pelvis to remove accumulations of gravel, sulphonamide crystals or inspissated pus. Removal of calculi or drainage of an obstructed renal pelvis by nephrostomy or pyelostomy may be necessary when ureteric catheterization is unsuccessful in restoring urinary secretion. Spinal and paravertebral anaesthesia to relieve glomerula vasospasm and renal decapsulation to reduce tension in upper nephron nephrosis have their advocates but are probably of limited value.

Apart from the types of anuria already mentioned there are certain well-recognized conditions which are fairly common in the practice of urology.

\section{Calculous Anuria}

Calculous anuria is usually due to a small moveable stone which has become impacted on its way down the ureter. It is often associated with a mass of gravel, blood clot or inspissated pus which forms a plug making a partial obstruction complete. The stone itself may be small in size and barely discernable in a radiogram. Complete anuria will result when the blockage is bilateral or when the opposite kidney is functionless because of disease, congenital absence or reflex inhibition. The onset is often insidious but generally follows repeated attacks of renal colic, the site of the pain indicating the side last obstructed. A complete urinary investigation is likely to reveal the cause and site of the obstruction, which must be relieved forthwith, either by ureteric catheterization, nephrostomy or ureterolithotomy.

\section{Reflex Anuria}

Reflex anuria is essentially a protective mechanism whereby, under certain circumstances, renal secretion is inhibited. It occurs particularly when a ureter is blocked, but may be reproduced experimentally by various stimuli of widely different kinds. It is brought about, in the first instance, by a local nervous reflex resulting in cortical ischaemia of the kidney from vasospasm and deviation of the blood flow through the kidney by means of the 'shunt' mechanism. There is evidence to support the view that this action can be prolonged indefinitely by hormonal influences Diminution or complete cessation of renal function is a common phenomenon after an attack of renatcolic and may last for several days. It must: therefore, be taken into account in the interpretation of intravenous pyelograms.

In some cases a normâl kidney may be inhibite by an obstruction of the opposite side (" crossed reflex anuria). Treatment is directed to removal of the cause, such as an obstruction in the uretef or a drainage tube pressing on the renal pedicle $\overrightarrow{0}$ after a renal operation. Attempts to interrupt the reflex arc by spinal or paravertebral anaesthesiff where the exciting cause is not obvious have beero made in cases of post-operative anuria and in the crush syndrome, but the results have not beent uniformly successful.

\section{Anuria Following Urethral Instrumentation}

Anuria may follow urethral instrumentation especially after severe urethral trauma in the presence of an infected urine. The rapid absorp $\overrightarrow{-}$ tion of bacteria from the raw surface will give ris $\Phi$ to septicaemia, the kidneys already damaged bF the effects of longstanding urinary obstruction bearing the brunt of the resulting systemic inf ${ }_{8} \mathrm{C}=$ tion. Prevention, apart from chemotherapy, con sists in gentleness when dilating urethral strictures $N$ especially when infection and signs of renal damâg are present. Other reminders of the absorptive powers of the urethra are provided by reports frono time to time of cases of mercurial poisoning fronf the use of mercurial solutions for bladder wash $\mathbb{\Omega}$ outs or cystoscopy (Page and Wilson, 194I), and ast the renal epithelium is always extensively damage 5 by the absorption of heavy metals, anuria tends to. be a prominent clinical feature.

Instances where anuria followed ureteris catheterization have also been reported. Here the mechanism appears to have been chiefly the effeci of reactionary oedema causing blockage of the ureter in association with absence of function o. possibly reflex inhibition of the other side. Lastly? after per-urethral resection of the prostate, anurie may develop from laking of the blood by hypo $\rightarrow$ tonic lotion used for irrigation, intravascula?. haemolysis giving rise to lower nephron nephrosis as in the 'transfusion kidney.'

\section{Injuries to the Genito-Urinary Organs}

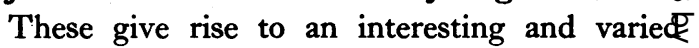
group of lesions which includes open wounds, subo cutaneous injuries (contusions of the kidney an testicle, ruptures of the ureter, bladder and urethra), surgical accidents (damage to the ureter or bladder during pelvic operations, perforations of the ureter, bladder or urethra by instruments of 
various kind used for diagnosis or treatment). These are mostly serious injuries often complicated by involvement of important neighbouring structures. They will present many diagnostic problems and as many of the cases will require operative treatment their management can only be properly carried out in hospital. The mechanism, the resulting lesion and the processes of repair will vary greatly at different levels in the urogenital tract.

\section{Kidney}

The kidney is usually injured by a force applied to its anterior surface through the soft tissues of the anterior abdominal wall which crushes it against the unyielding structures posteriorly. The injury is often trivial, the direction rather than the severity of the force determining the resulting rena! lesion. This is almost invariably a deep fissure of the parenchyma radiating out from the hilum and involving the softer medulla more extensively than the firmer cortex. This gives rise to intrapelvic haemorrhage manifested by haematuria or, less commonly, to perirenal haemorrhage with the formation of a haematoma around the kidney. Haemorrhage will cease spontaneously and spontaneous cure with a variable amount of scarring at the site of the lesion will take place in approximately 90 per cent. of these injuries. In the remaining Io per cent. operative interference will be necessary, either to control severe haemorrhage or to deal with associated intraperitoneal lesions such as ruptured spleen, liver or gut.

Treatment is, therefore, in the first place, always expectant, but in view of the possibility that nephrectomy may have to be urgently undertaken, it is essential to establish the presence of a sound kidney on the opposite side, either by intravenous or retrograde pyelography, at the earliest possible moment. This becomes a matter of great practical importance when it is remembered that an enlarged kidney is more vulnerable to trauma than a normal one, and that a common cause of renal enlargement is compensatory hypertrophy due to disease or congenital absence on the opposite side. All patients treated conservatively should be kept in hospital at least three weeks as repair of renal parenchyma is a slow process and the danger of secondary haemorrhage is not past until the end of that time. It is always advisable to make certain by means of intravenous pyelography that renal function has returned to normal and that there is no obstruction to the renal pelvis by scarring before returning the patient to work.

\section{Ureter}

The ureter, owing to its deep position in the body, is rarely injured except by the surgeon. He may accidentally clamp or divide it when dealing 2 with large or adherent pelvic tumours or when re- $\frac{\widehat{\phi}}{\mathrm{\phi}}$ secting the pelvic colon, or may perforate it from within by means of ureteric catheters, by over $\rightarrow$ vigorous dilatation of a ureteral stricture or bys forcible attempts to extract calculi with wireo ' baskets ' or similar instruments. Treatment con-듬 sists of immediate end-to-end suture of a severed $\overline{\bar{c}}$. ureter if the injury is recognized at the time. $\mathbb{Q}$ Where some time has elapsed it is sometimeso possible to anastomose the damaged ureter to the ${ }^{\infty}$ bladder or bowel, although in most cases, especially $\vec{\circ}$ where a urinary fistula has developed, the results $\overrightarrow{\vec{\omega}}$ of conservative operations are unsatisfactory. $\omega$ When the kidney of the opposite side is sound, nephrectomy is usually the best form of treatment. 3 .

\section{Bladder}

The bladder may be ruptured from without by $\frac{\omega}{\sigma}$ missiles, kicks or blows or by violent lateral compression in association with injuries of the pelvic girdle. It may also be accidentally incised by the surgeon in performing any pelvic operation, $\vec{T}$ especially when the pre-operative precaution of $\frac{\mathbb{O}}{O}$ emptying the bladder by means of a catheter has been omitted. Intravesical surgical manipulations of any kind, rough instrumentation, over-diste $8-\overrightarrow{0}$ sion, excessive diathermy, etc., may perforate actually burst the bladder from within. Spos? taneous rupture of the over-distended bladder cक्ष readily occur when its wall has been weakened by disease, especially ulceration of any kind, although in some of the cases reported (Beresford-Jones, I94I, and others) the wall of the viscus was ap- $\varnothing$ parently normal before rupture. The diagnosis of $\overrightarrow{0}$ ruptured bladder is notoriously difficult chiefly 3 because typical symptoms and signs are late in appearing and are often effectively masked in the earlier stages by shock and associated injuries.음 Cystoscopy and cystography are valuable aids to diagnosis, but whenever there is any doubt ex- $\frac{\sigma}{3}$ ploration of the bladder should be undertaken as: soon as conditions permit. Suture of the rent ando adequate drainage, not only of the bladder but also of the paravesical tissues, are the main princip's of treatment.

\section{Urethra}

The urethra may be ruptured completely or partially, the latter type of injury being due as a rule to the surgeon causing 'false passages' by burrowing with the point of an instrument either in the spongy tissue of the bulb or in the sub-o mucosa of the prostatic urethra. Such injuries may cause severe haemorrhage, clot retention, infection (periurethral suppuration) or extravasation of urine.

Complete ruptures are found at two classica 
sites, either just below the triangular ligament where they are caused by falls astride or other injuries to the perineum, or immediately above the triangular ligament in association with fractures of the pelvic girdle. At either site there is wide separation of the torn ends and reconstructive surgery at the earliest possible moment is essential in order to secure union without excessive scar formation. The distal type of rupture is best dealt with by end-to-end suture from the perineum, the proximal by retrograde catheterization and weight extension. Urinary diversion by suprapubic cystostomy is necessary in both cases while healing is taking place.

\section{Male Genitalia}

The male genitalia, owing to their exposed position, are very vulnerable. Typical lesions are the result of contusions, open wounds or avulsion injuries. Because of the great vascularity of the parts, genital wounds bleed profusely. In addition, nervous shock is often a striking phenomenon in these cases, so that the patient is frequently found in a profound state of collapse out of all proportion to the severity of the local lesion.

Treatment should in the first place be confined to the control of haemorrhage, the relief of tension by evacuation of retained blood clot and the provision of free drainage. No tissue should be cut away unless it is frankly non-viable. Healing is rapid and much can be done to remedy defects subsequently by plastic surgery. The familiar contusion of the testicle may be caused by kicks, crushes *or missiles; the careless tapping of $a \frac{\$}{\Phi}$ hydrocoele may produce the same result. The $\propto$ resulting haematoma when not too large will sub- $c$ side rapidly with rest elevation, cold applications, $\overrightarrow{\vec{s}}$ etc. More severe injuries will often rupture the testis and produce a large haematoma within the $\frac{\overline{0}}{-}$ tunica vaginalis (haematocoele). Operative treat- $\overline{\bar{c}}$. ment is indicated in the latter type of injury with $\vec{\varnothing}$ the object of turning out the clot and suturing the rent in the visceral layer of the tunica in order to ${ }^{\infty}$ prevent subsequent atrophy of the testis.

Contusions of the penis usually affect the erect $\frac{\vec{\omega}}{\omega}$ organ (e.g. ' faux pas de coit'), and may result in $\stackrel{\circ}{\circ}$ dislocation, fracture or haematoma within thee sheath of the corpus cavernosum. When the 3 . latter type of injury is severe removal of the cloti and suture of the sheath may be indicated. $\dot{\omega}$ Avulsion of the genitalia may occur in factories $\vec{\sigma}$ when the patient's clothes are caught up in rotating $0_{0}^{\circ}$ belts.

\section{Paraphimosis}

Paraphimosis is treated by manual reduction or incision of the constriction with subsequent circumcision to enlarge the preputial opening after the oedema has subsided. Strangulation by rubberc bands or metal rings applied to the flaccid orgain by sexual perverts or to control enuresis will often? cause gangrene before the constricting agent can be removed and subsequent skin grafting may be necessary.

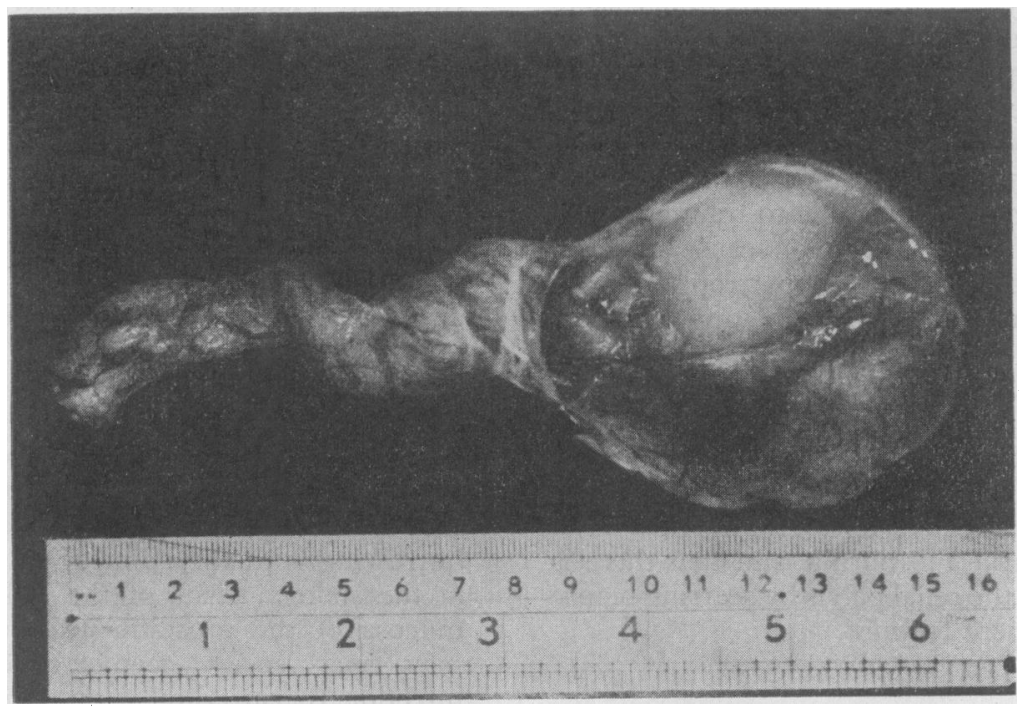

FIG. 2.-Longstanding torsion of spermatic cord; note secondary hydrocele and atrophy of testis 


\section{Genital Torsions}

Genital torsions are commoner than is generally supposed and may affect the spermatic cord, the testis or the stalked hydatid of Morgagni. Twists of the spermatic cord are apt to occur during late adolescence and are perhaps the commonest cause of testicular atrophy (Fig. 2). The aetiology is unknown, but excessive mobility of the testicle within the scrotum undoubtedly plays an important part. The onset is generally characteristic, with sudden pain and moderate swelling associated with mild fever. In spite of the fact that the entire testicle is enlarged the condition is often mistaken for epididymitis and treated as such, and when bilateral involvement occurs this may have serious consequences, leading not only to.sterility but to eunuchodism. As degenerative changes in the testicular epithelium will commence within a. few hours of torsion it is imperative that exploration be undertaken immediately, hence the supreme importance of early diagnosis. Operative treatment consists of untwisting the torsion, everting the tunica and stitching the edges to the back of the scrotum to prevent further attacks. As the condition is frequently bilateral it is usual to fix both testicles through a midline scrotal-splitting incision.

Torsion of the testis alone may occur when the mesorchium is long: This may take place within the tunica when the testis is in the scrotum, but is more often found in association with imperfect descent. Torsion of an intra-abdominal testis has been mistaken for an acute abdomen, and nearly 50 per cent. of such organs are the site of tumour formation. Torsion of the hydatid of Morgagni, though not serious in itself, is often followed by testicular pain of a persistent character (" testicular neuralgia') which is not always relieved by removal of the subsequent cyst or even by orchidectomy.

\section{Spontaneous Perirenal Haematoma}

Spontaneous perirenal haematoma is a con- $\frac{3}{2}$ dition which always arouses great clinical interest $\stackrel{\varrho}{c}$ because of its dramatic features. Spontaneous $\vec{\Rightarrow}$ bleeding may take place into the perirenal or sub- $\stackrel{9}{+}$ capsular spaces as a result of renal or adrenal $\bar{C}$ disease, diseases of the renal blood vessels or blood $\overline{\bar{N}}$. dyscrazias. No determining cause can be found in $\frac{\bar{m}}{\overline{0}}$. about 25 per cent. of the cases but trauma must, $\stackrel{\varnothing}{\varrho}$ of course, be carefully excluded before any case of renal haemorrhage is classified under this heading. $\vec{P}$

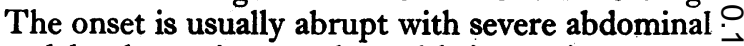
and lumbar pain, together with increasing symp- $\vec{\omega}$ toms and signs of internal haemorrhage. The $\stackrel{\sigma}{\circ}$ differential diagnosis from other forms of $a b-\frac{D}{2}$ dominal emergency is not easy. The mortality is 3 . high because of the frequent delay in recognizing $\underset{\infty}{\sim}$ the condition. 'Treatment usually takes the form $\omega$ of rapid nephrectomy although bleeding can $\vec{\sigma}$ sometimes be controlled by more conservative $\varnothing_{0}^{\circ}$ measures such as packing, ligature or suture.

\section{Sevére Renal Haematuria}

Severe renal haematuria may be caused by $\frac{\pi}{0}$ tumours of the kidney or renal pelvis, hypertensive granular red kidney or renal purpura (essential renal haematuria). Where bleeding from any of $\vec{\theta}$ these conditions is severe enough to threaten the on patient's life, nephrectomy may become a matte of extreme urgency and the objects of an in vestigation of such cases should be not only to reveal the site and the cause of the bleeding but also the state of the kidney on the opposite side.

\section{Açute Pyonephrosis}

An acute pyonephrosis arises when a ful- $\frac{0}{3}$ minating infection with pyogenic bacteria takes place in an obstructed renal pelvis. If this condition is not promptly relieved by drainage or $\bar{\partial}$ nephrectomy there is always a very real danger of 3 a serious and often fatal septicaemia.

\section{BIBLIOGRAPHY}

BERESFORD-JONES, A. B. (1941), Brit. F. Surg., 24, 54. BULL, G. M., JOEKES, A. M., and LOWE, K. G. (1949), Lancet, ii, 229.

BYWATERS, E. G. L., ạnd BEALI, D. (1941), Brit.med. F., i, 427.
PAGE, B. H., and WILSON, C. (1940), Lancet, i, 640.

TRUETA, J., BARCLAY, A. E., DANIEL, P. M., FRANKLIN, K. J., and PRICHARD, M. M. L. (1947), 'Studies of the I Renal Circulation,' Blackwell Scientific Publications, Oxford. 